\title{
APPLICATIONS OF THE LIE THEORY OF EXTENDED GROUPS IN HAMILTONIAN MECHANICS: THE OSCILLATOR AND THE KEPLER PROBLEM
}

\author{
P. G. L. LEACH \\ (Received 24 September 1980) \\ (Revised 22 December 1980)
}

\begin{abstract}
The method of the Lie theory of extended groups has recently been formulated for Hamiltonian mechanics in a manner which is consistent with the results obtained using the Newtonian equation of motion. Here the method is applied to the three-dimensional time-independent harmonic oscillator and to the classical Kepler problem. The expected constants of motion are obtained. Previously unobserved relations between generators and invariants are also noticed.
\end{abstract}

\section{Introduction}

There is, it would seem, a never-ending search for methods which provide a way of determining symmetries and invariants for dynamical systems. Two useful methods are Noether's theorem (in its various forms) and the method of the Lie theory of extended groups. The present application of both methods is to test systems for the presence of exact symmetries and, if they exist, to determine the associated constants of the motion. It would not greatly surprise us if in the future they were, in some sense, used to determine approximate symmetries, for example, of an adiabatic type.

The two methods are based on the concept of an invariance under an infinitesimal transformation of the dynamical variables. For Noether's theorem, the object which is left invariant is the Action Integral and, for the Lie method, it is the equation(s) of motion. The latter method is less restrictive than the

OCopyright Australian Mathematical Society 1981 
former and provides a greater number of invariants and/or allows a more general class of problem to be treated.

Before proceeding further it is proper that we define the type of infinitesimal transformation about which we speak. We are dealing with point transformations only. As an example of the greater generality of the Lie method we cite a one-dimensional linear system. Allowing point transformations only, Noether's theorem yields five generators of symmetry whereas the Lie method provides eight. However, the more serious failings of Noether's theorem occur when multi-dimensional systems are studied. It does not provide the Jauch-HillFradkin tensor for the harmonic oscillator nor the Runge-Lenz vector for the classical Kepler problem. The Lie method does [5], [6].

To repair this deficiency in Noether's theorem, the use of velocity-dependent transformations has been proposed [1], [4]. Certainly the constants mentioned above satisfy the equations obtained with the more general type of transformation. Unfortunately, the wider class of admissible transformations results in an infinite number of symmetries for which no systematic method of determination exists. The same fault applies to the Lie method if the inclusion of velocity-dependent transformations is allowed. However, the inclusion is not necessary for the Lie method since all the useful invariants may be found with coordinate-dependent transformations only. As far as we are aware, invariants of value in describing the motion are either linear or quadratic in the velocities (momenta). It is these invariants which we term useful here. The difficulties associated with the use of velocity-dependent transformations in Noether's theorem are delineated more fully in the Appendix.

Until recently [7], the application of the Lie method in mechanics has been to the Newtonian equation of motion whereas Noether's theorem may be applied in either a Lagrangian or Hamiltonian context. Once the Newtonian results were obtained, the results could be translated into Hamiltonian form [2], but it seemed to us to be a messy approach. As many problems occur in a Hamiltonian framework, we judged it better to formulate the Lie method in the Hamiltonian framework in such a way that the results obtained would be consistent with the results for the corresponding Newtonian system when it exists. The formulation turned out to be very straightforward and we simply quote the results here. The operator

$$
Y(\mathbf{q}, \mathbf{p}, t)=\xi(\mathbf{q}, t) \partial / \partial t+\eta_{i}(\mathbf{q}, t) \partial / \partial q_{i}+\zeta_{i}(\mathbf{q}, \mathbf{p}, t) \partial / \partial p_{i}
$$

is a generator of a one-parameter Lie group for a Hamiltonian $H(\mathbf{q}, \mathbf{p}, t)$ with equations of motion

$$
\dot{q}_{i}-\partial H / \partial p_{i}=0 \quad \text { and } \quad \dot{p}_{i}+\partial H / \partial q_{i}=0
$$


provided the action of the first extension of $Y$ on equations (1.2) gives zero whenever equations (1.2) are satisfied. The first extension is

$$
Y^{(1)}(\dot{\mathbf{q}}, \dot{\mathbf{p}}, \mathbf{q}, \mathbf{p}, t)=Y(\mathbf{q}, \mathbf{p}, t)+\eta_{i}^{(1)}(\mathbf{q}, \mathbf{p}, t) \partial / \partial \dot{q}_{i}+\zeta_{i}^{(1)}(\mathbf{q}, \mathbf{p}, t) \partial / \partial \dot{p}_{i},
$$

where we have specifically written $\eta_{i}^{(1)}$ and $\zeta_{i}^{(1)}$ as functions of $\mathbf{q}, \mathbf{p}$ and $t$ to indicate that whenever $\dot{q}_{j}$ appears it is replaced by $\partial H / \partial p_{j}$ and $\dot{p}_{j}$ by $-\partial H / \partial q_{j}$. Thus

$$
\eta_{i}^{(1)}=\frac{\partial \eta_{i}}{\partial t}+\frac{\partial H}{\partial q_{j}} \frac{\partial \eta_{i}}{\partial q_{j}}-\frac{\partial H}{\partial p_{i}}\left(\frac{\partial \xi}{\partial t}+\frac{\partial H}{\partial p_{j}} \frac{\partial \xi}{\partial q_{j}}\right)
$$

and

$$
\zeta_{i}^{(1)}=\frac{\partial \zeta_{i}}{\partial t}+\frac{\partial H}{\partial p_{j}} \frac{\partial \zeta_{i}}{\partial q_{j}}-\frac{\partial H}{\partial q_{j}} \frac{\partial \zeta_{j}}{\partial p_{j}}+\frac{\partial H}{\partial q_{i}}\left(\frac{\partial \xi}{\partial t}+\frac{\partial H}{\partial p_{j}} \frac{\partial \xi}{\partial q_{j}}\right) .
$$

When the set of generators $\left\{Y_{i}\right\}$ is determined, the associated invariants are obtained as solutions to the pair of equations

$$
Y_{i}(\mathbf{q}, \mathbf{p}, t) I(\mathbf{q}, \mathbf{p}, t)=0
$$

and

$$
\frac{d}{d t}\{I(\mathbf{q}, \mathbf{p}, t)\}=0 .
$$

This is the main drawback with the Lie method in comparison with Noether's theorem as the latter gives the invariant immediately. There are some instances in which the integration of equations (1.6) and (1.7), or their equivalents in the Newtonian picture, is not necessary. The time-dependent one-dimensional harmonic oscillator [3] may be taken as an example. The generators for that problem may be converted to the generators for the corresponding time-independent oscillator by a common transformation. Applying the inverse transformation to the oscillator invariants, the invariants for the time-dependent problem are obtained immediately.

The plan of this paper is as follows. In Section 2 the generators for the class of Hamiltonian systems to which the oscillator and the Kepler problem belong are shown to have a particular form. This simplifies the algebra when the two problems are considered in Sections 3 and 4.

\section{Possible form of the generator}

Suppose we have a Hamiltonian for which the equations of motion have the form

$$
\dot{q}_{i}-f_{i j}(t) p_{j}=0
$$


and

$$
\dot{p}_{i}+g_{i}(\mathbf{q}, t)=0,
$$

which is commonly the case when the basis is cartesian. The matrix $\left[f_{i j}\right]$ is regular and may, without loss of generality, be taken as symmetric, although this is not necessary. Applying the first extension of the operator $Y$ given in equation (1.3) to equations (2.1) and (2.2) we have

$$
\eta_{i}^{(1)}-Y(\mathbf{q}, \mathbf{p}, t) f_{i j}(t) p_{j}=0
$$

and

$$
\zeta_{i}^{(1)}+Y(\mathbf{q}, \mathbf{p}, t) g_{i}(\mathbf{q}, t)=0 .
$$

On rearranging equation (2.3) we obtain

$$
\zeta_{i}=f^{i k} \eta \eta_{k}^{(1)}-\xi f^{i k}\left(\partial f_{k j} / \partial t\right) p_{j}
$$

where $\left[f^{i j}\right]$ is the inverse of $f_{i j}$, that is,

$$
f^{i k} f_{k j}=\delta_{j}^{i}
$$

This expression for $\zeta_{i}$ is substituted into equation (2.4). The terms of third order in the momenta yield

$$
\frac{\partial^{2} \xi}{\partial q_{i} \partial q_{j}}=0
$$

and so

$$
\xi(\mathbf{q}, t)=a(t)+b_{i}(t) q_{i} .
$$

Collecting and rearranging the terms of second order in the momenta,

$$
\begin{aligned}
\left\{f^{i k} f_{m n} f_{j l} \frac{\partial^{2} \eta_{k}}{\partial q_{j} \partial q_{n}}-2 \delta^{i} m f_{n l} \frac{\partial^{2} \xi}{\partial q_{n} \partial t}-\delta_{m}^{i} \frac{\partial f_{j l}}{\partial t} \frac{\partial \xi}{\partial q j}-f_{j l} f^{i k} \frac{\partial f_{k m}}{\partial t} \frac{\partial \xi}{\partial q_{j}}\right\} \\
\times\left\{\delta_{u l} \delta_{v m}+\delta_{v l} \delta_{u m}\right\}=0,
\end{aligned}
$$

where equation (2.6) has also been used. For equation (2.9), it is obvious that $\eta$ is at most quadratic in q. From the particular case $f_{i j}=\delta_{i j}$ we get

$$
\eta_{i}(\mathbf{q}, t)=\dot{b}_{j} q_{j} q_{i}+c_{i j}(t) q_{j}+d_{i}(t) .
$$

There is no point, at this stage, in considering the coefficients of first and zero-th order powers of $p$ since these contain the functions $g_{i}(\mathbf{q}, t)$. Had we allowed the $f_{i j}(t)$ to be functions of $q$ as well, equation (2.10) would have been much more complex. Such dependence would occur when curvilinear coordinates are used and may, as a separate topic, be worthy of discussion. However, the cartesian form which we are using here is sufficient for the present purpose. To round off this section we complement equations (2.8) and (2.10) 
with the expression for $\zeta_{i}(\mathbf{q}, \mathbf{p}, t)$ for the case when $f_{i j}$ is $\delta_{i j}$. It is

$$
\zeta_{i}(\mathbf{q}, \mathbf{p}, t)=-\dot{a} p_{i}+\ddot{b}_{j} q_{j} q_{i}+\dot{b}_{j} p_{j} q_{i}-b_{j} p_{j} p_{i}+\dot{c}_{i j} q_{j}+c_{i j} p_{j}+\dot{d}_{i} .
$$

\section{Harmonic oscillator}

The three-dimensional time-independent harmonic oscillator has the Hamiltonian

$$
H=\frac{1}{2}\left(\mathbf{p}^{2}+\mathbf{q}^{2}\right)=\frac{1}{2}\left(p_{i} p_{i}+q_{i} q_{i}\right), \quad i=1,3 .
$$

Equations (2.2) and (2.3) become

$$
\eta_{i}^{(1)}-\zeta_{i}=0 \quad \text { and } \quad \zeta_{i}^{(1)}+\eta_{i}=0 .
$$

Corresponding to the development in the previous section, the first of (3.2) gives $\zeta_{i}$ in terms of $\eta_{i}, \xi$ and their derivatives. Using the second of (3.2), the general formulae obtained for $\zeta_{i}, \eta_{i}$ and $\xi$, and separating by coefficients of powers of $p$ and $q$ in turn, we obtain the following set of ordinary differential equations

$$
\left\{\begin{array}{l}
2 \dot{a} \delta_{i k}+\ddot{c}_{i k}=0, \quad \ddot{a} \delta_{i k}-2 \dot{c}_{i k}=0, \\
\ddot{b}_{i}+b_{i}=0, \quad \text { and } \quad \ddot{d}_{i}+d_{i}=0 .
\end{array}\right\}
$$

The solution of this set of equations is trivial and results in the following expressions for $\xi, \eta$ and $\zeta$ :

$$
\begin{aligned}
\xi(\mathbf{q}, t)= & A+B \sin 2 t+C \cos 2 t+\left(D_{j} \sin t+E_{j} \cos t\right) q_{j}, \\
\eta_{i}(\mathbf{q}, t)= & \left(D_{j} \cos t-E_{j} \sin t\right) q_{j} q_{i}+F_{i k} q_{k} \\
& +(B \cos 2 t-C \sin 2 t) q_{i}+G_{i} \sin t+H_{i} \cos t \\
\zeta_{i}(\mathbf{q}, \mathbf{p}, t)= & -(B \cos 2 t-C \sin 2 t) p_{i}-\left(D_{j} \sin t+E_{j} \cos t\right) q_{j} q_{i} \\
& +\left(D_{j} \cos t-E_{j} \sin t\right) p_{j} q_{i}-\left(D_{j} \sin t+E_{j} \cos t\right) p_{j} p_{i} \\
& -2(B \sin 2 t-C \cos 2 t) q_{i}+F_{i j} p_{j}+G_{i} \cos t-H_{i} \sin t
\end{aligned}
$$

In all, for the three-dimensional problem, the generator contains twenty-four constants of integration giving twenty-four linearly independent generators. In passing, we note that for the corresponding $n$-dimensional system there are $n^{2}+4 n+3$ constants and so an equal number of generators (compare with [1]). From the expressions given in equations (3.4) to (3.6) we may write down the individual generators. These are

$$
\begin{aligned}
& Y_{1 i}=\sin t \partial / \partial q_{i}+\cos \partial / \partial p_{i}, \quad i=1,3 \\
& Y_{2 i}=\cos t \partial / \partial q_{i}-\sin t \partial / \partial p_{i}, \quad i=1,3 \\
& Y_{3}=\partial / \partial t \\
& Y_{4}=\sin 2 t \partial / \partial t+q_{j} \cos 2 t \partial / \partial q_{j}-\left(2 q_{j} \sin 2 t+p_{j} \cos 2 t\right) \partial / \partial p_{j}
\end{aligned}
$$




$$
\begin{array}{rlr}
Y_{5}= & \cos 2 t \partial / \partial t-q_{j} \sin 2 t \partial / \partial q_{j}-\left(2 q_{j} \cos 2 t-p_{j} \sin 2 t\right) \partial / \partial p_{j} \\
Y_{6 i}= & q_{i} \sin t \partial / \partial t+q_{i} q_{j} \cos t \partial / \partial q_{j} \\
& -\left(q_{i} q_{j} \sin t-p_{i} q_{j} \cos t-p_{i} p_{j} \sin t\right) \partial / \partial p_{j}, \quad i=1,3 \\
Y_{7 i}= & q_{i} \cos t \partial / \partial t-q_{i} q_{j} \sin t \partial / \partial q_{j} \\
& -\left(q_{i} q_{j} \cos t+p_{i} q_{j} \sin t+p_{i} p_{j} \cos t\right) \partial / \partial p_{j}, & i=1,3 \\
Y_{8 i j}= & q_{j} \partial / \partial q_{i}+q_{i} \partial / \partial q_{j}+p_{j} \partial / \partial p_{i}+q p_{i} \partial / \partial p_{j}, \quad i, j=1,3 \\
Y_{9 i j}= & q_{j} \partial / \partial q_{i}-q_{i} \partial / \partial q_{j}+p_{j} \partial / \partial p_{i}-p_{i} \partial / \partial p_{j}, \quad i \neq j=1,3 .
\end{array}
$$

Note that the generators for $Y_{8 i j}$ and $Y_{9 i j}$ are obtained from the symmetric and antisymmetric parts of $F_{i j}$, respectively.

We do not intend to discuss the group structure in detail here as it corresponds with the results given by Prince and Eliezer [5] for the time-dependent system, that is, the group is $\mathrm{Sl}(3+2, R)$. Furthermore, we shall not explore all the invariants in detail. Of the generators with time-dependent coefficients, $Y_{1 i}$ and $Y_{2 i}$ are associated with the invariants which represent the initial conditions. The others are not so informative about the motion.

However, we do wish to make some comment about the generators $Y_{3}, Y_{8 i j}$ and $Y_{9 i j}$. The antisymmetric generator $Y_{9 i j}$ produces the invariants

$$
I_{9 i j 1}=q_{i} p_{j}-q_{j} p_{i}, \quad i \neq j,
$$

and

$$
I_{9 i j 2}=q_{i}^{2}+q_{j}^{2}+p_{i}^{2}+p_{j}^{2}, \quad i \neq j,
$$

there being three of each type. The first type consists of the components of angular momentum. To each component of the angular momentum there is one invariant of the second type which represents a hypersphere in the four-dimensional subspace of phase space given by the coordinates $\left(q_{i}, q_{j}, p_{i}, p_{j}\right)$. This hypersphere is invariant under rotations. The symmetric generator $Y_{8 i j}$ gives rise to what might be described as the hyperbolic counterpart of the invariant hypersphere. The two constants may be considered to be the orthogonal projections of a pair of rotating (hyper-) hyperboloids specified respectively by

$$
f_{1}\left(q_{i}, q_{j}, p_{i}, p_{j}\right)=\left(q_{i}^{2}-q_{j}^{2}\right)-\left(p_{i}^{2}-p_{j}^{2}\right)
$$

and

$$
f_{2}\left(q_{i}, q_{j}, p_{i}, p_{j}\right)=q_{i} p_{i}-q_{j} p_{j} \text {. }
$$

So we see that the symmetric generator is not of use to describe the motion whereas the antisymmetric operator gives rise to the angular momentum and, when summed, the energy, both of which are useful quantities. 
We have purposely left the simplest generator, that of time-translations, to the last as it displays a richness of detail which is generally overlooked. We suspect the reason for this lies in the consequence of the use of Noether's theorem as in that context this generator gives rise to the conserved energy alone. However, we are working within the scheme of the Lie method and shall display a far richer result. We recall that an invariant associated with a generator satisfies the dual conditions

$$
Y(\mathbf{q}, \mathbf{p}, t) I(\mathbf{q}, \mathbf{p}, t)=0 \quad \text { and } \quad . \dot{I}(\mathbf{q}, \mathbf{p}, t)=0 .
$$

For $Y_{3}$ the associated Lagrange's system for the first of (3.20) is

$$
d t / 1=d q_{i} / 0=d p_{i} / 0, \quad i=1,3,
$$

whence, trivially,

$$
u_{i}=q_{i} \quad \text { and } \quad v_{i}=p_{i}
$$

Rewriting the second of (3.20) in terms of the $u_{i}$ and $v_{i}$, we have

$$
d u_{i} / d u_{j}=v_{i} / v_{j}, \quad d v_{i} / d v_{j}=u_{i} / u_{j} \quad \text { and } \quad d v_{i} / d u_{j}=-u_{i} / v_{j} \text {. }
$$

Taking the third of (3.23),

$$
u_{i} d u_{j}+d v_{i} v_{j}=0
$$

and adding to it the same with $i$ and $j$ interchanged, we obtain

$$
d\left(u_{i} u_{j}+v_{i} v_{j}\right)=0
$$

Integration of equation (3.25) gives the Jauch-Hill-Fradkin tensor

$$
A_{i j}=q_{i} q_{j}+p_{i} p_{j}
$$

Half the trace of $A_{i j}$ gives the energy. The first two of equation (3.23) may be rewritten as

$$
d u_{i} v_{j}-d u_{j} v_{i}=0 \quad \text { and } \quad u_{i} d v_{j}-u_{j} d v_{i}=0
$$

When these are added and integrated we have the angular momentum tensor

$$
L_{i j}=q_{i} p_{j}-q_{j} p_{i} \text {. }
$$

The Jauch-Hill-Fradkin tensor does not arrive from the application of Noether's theorem. The reason for this stems from two facts. The first is that only one invariant is found per generator and the $\partial / \partial t$ generator, by not containing the coordinates specifically, can be regarded as a diagonal operator which, in combination with a diagonal Lagrangian (Hamiltonian), gives a diagonal invariant, the trace of the tensor. The second is that the only other possible source could be one of the time independent generators $Y_{8 i j}$ and $Y_{9 i j}$, actually their Noether equivalents, and neither of these provides the tensor when Noether's theorem is used. 
It must be emphasized that we are not promoting the use of the generator, $\partial / \partial t$, to obtain all of the time-independent first integrals. This might require considerable ingenuity, rather more, in fact, than is required when the generator directly associated with the particular first integral is used. What we do wish to demonstrate is that, in the Lie method, such first integrals are implied in the generator of time translations.

\section{Classical Kepler problem}

The Hamiltonian for the classical Kepler problem is

$$
H=\frac{1}{2} p^{2}-\mu / r
$$

and, in light of the development in Section 2, we take cartesian coordinates. Equations (2.2) and (2.3) become

$$
\eta_{i}^{(1)}-\zeta_{i}=0 \quad \text { and } \quad \zeta_{i}^{(1)}+\mu \eta_{j}\left(\delta_{i j} / r^{3}-3 q_{i} q_{j} / r^{5}\right)=0 .
$$

Following the procedure used in Section 3 we find that

$$
\left.\begin{array}{l}
a(t)=A t+B, \quad b_{i}(t)=0, \\
C_{i j}(t)=\frac{2}{3} A \delta_{i j}+C_{i j} \quad \text { and } \quad d_{i}(t)=0,
\end{array}\right\}
$$

where the constant matrix $\left[C_{i j}\right]$ is skew-symmetric. The generators of oneparameter Lie groups are

$$
\begin{aligned}
Y_{1} & =\partial / \partial t \\
Y_{2} & =t \partial / \partial t+\frac{2}{3} q_{j} \partial / \partial q_{j}-\frac{1}{3} p_{j} \partial / \partial p_{j}, \\
Y_{3 i j} & =q_{i} \partial / \partial q_{j}-q_{j} \partial / \partial q_{i}+p_{i} \partial / \partial p_{j}-p_{j} \partial / \partial p_{i}, \quad i \neq j=1,3,
\end{aligned}
$$

a total of five in all since $Y_{3}$ is skew-symmetric.

Starting with $Y_{3 i j}$, the associated Lagrangian system is

$$
d t / 0=d q_{i} / q_{j}=d q_{j} /\left(-q_{i}\right)=d p_{i} / p_{j}=d p_{j} /\left(-p_{i}\right), \quad i \neq j,
$$

for which

$$
\left.\begin{array}{l}
u_{1}=t, \quad u_{2 i j}=q_{i}^{2}+q_{j}^{2}, \\
v_{1 i j}=p_{i}^{2}+p_{j}^{2} \quad \text { and } \quad v_{2 i j}=q_{i} p_{j}-q_{j} p_{i} .
\end{array}\right\}
$$

From (4.8) and the equations of motion we have

$$
\begin{gathered}
d u_{2 i j} / d u_{1}=2\left(q_{i} p_{i}+q_{j} p_{j}\right), \quad d v_{1 i j} / d u_{1}=-2\left(q_{i} p_{i}+q_{j} p_{j}\right) \mu / r^{3} \\
\text { and } \quad d v_{2 i j} / d u_{1}=0 .
\end{gathered}
$$


From the third of equation (4.9) it follows immediately that $v_{2 i j}$ is a first integral, in fact the angular momentum,

$$
L_{i j}=q_{i} p_{j}-q_{j} p_{i} .
$$

Combining the first two of (4.9),

$$
d v_{1 i j}+\left(\mu / r^{3}\right) d u_{2 i j}=0 .
$$

Summing this over the three permissible values of $i$ and $j$ and returning to the canonical coordinates,

$$
d\left(2 \mathrm{p}^{2}\right)+4 \mu d r / r^{2}=0
$$

which, on integration, gives the conserved energy,

$$
E=H=\frac{1}{2} \mathbf{p}^{2}-\mu / r
$$

For $Y_{2}$ we have

$$
d t / t=\left(d q_{j}\right) /\left(\frac{2}{3} q_{j}\right)=\left(d p_{j}\right) /\left(-\frac{1}{3} p_{j}\right), \quad j=1,3,
$$

and so

$$
u_{i}=q_{i} t^{-2 / 3} \quad \text { and } \quad v_{i}=p_{i} t^{1 / 3}, \quad i=1,3 .
$$

From (4.15) and the equations of motion we have

$$
\frac{d u_{i}}{d u_{j}}=\frac{3 v_{i}-2 u_{i}}{3 v_{j}-2 u_{j}} \quad \text { and } \quad \frac{d v_{i}}{d u_{j}}=\frac{v_{i}-3 \mu u_{i} P^{-3}}{3 v_{j}-2 u_{j}}
$$

where

$$
P^{2}=u_{k} u_{k}
$$

There are three possible solutions to equations (4.16), the components of the Runge-Lenz vector,

$$
R_{i}=u_{i} v_{j} v_{j}-u_{j} v_{i} v_{j}-\mu u_{i} / P=q_{i} p_{j} p_{j}-q_{j} p_{i} p_{j}-\mu q_{i} / r .
$$

That the energy and angular momentum cannot be solutions may be verified by allowing $Y_{2}$ to act on each and noting that the result is non-zero.

The associated Lagrange system for $Y_{1}$ is

$$
d t / 1=d q_{i} / 0=d p_{i} / 0, \quad i=1,3,
$$

and so

$$
u_{i}=q_{i} \quad \text { and } \quad v_{i}=p_{i}, \quad i=1,3 .
$$

From (4.2) and the equations of motion we have the relations

$$
d u_{j} / d u_{i}=v_{j} / v_{i}, \quad d v_{j} / d v_{i}=u_{j} / u_{i} \quad \text { and } \quad d v_{j} / d u_{i}=-\left(\mu u_{j}\right) /\left(r^{3} v_{i}\right) .
$$


The first and second of these may be combined to give

$$
d\left(u_{i} v_{j}-u_{j} v_{i}\right)=0,
$$

which yields the conserved angular momentum tensor

$$
L_{i j}=q_{i} p_{j}-q_{j} p_{i} \text {. }
$$

Using the third of (4.21) in diagonal form and summing we have

$$
v_{i} d v_{i}-\mu d(1 / r)=0
$$

which integrates to give the conserved energy

$$
E=H=\frac{1}{2} p^{2}-\mu / r .
$$

Now we take the third of (4.21) in its general form, multiply by $u_{(i / f)}$ and sum on the repeated index to obtain

$$
u_{i} v_{i} d v_{j}-\mu u_{j} d(1 / r)=0 \quad \text { and } \quad u_{j} v_{i} d v_{i}-\mu u_{j} d(1 / r)=0,
$$

which may be combined to give

$$
u_{i} d\left(v_{j} v_{j}\right)-u_{j} v_{j} d v_{i}-\mu u_{i} d(1 / r)=0 .
$$

Employing the first and third of equation (4.21), this may be rewritten as

$$
d\left(u_{i} v_{i} v_{j}-u_{j} v_{j} v_{i}-\mu u_{i} / r\right)=0,
$$

which integrates immediately to the components of the Runge-Lenz vector (4.18).

\section{Comment}

In this short note we have illustrated the use of the method of the Lie theory of extended groups in the context of Hamilton's equations of motion. We have demonstrated that the method may be applied in a self-contained way and yield results consistent with those obtained by the more usual application of the method to the Newtonian equations of motion. This consistency is achieved by restricting the choice of infinitesimal transformations to point transformations. The restriction is not unreasonable since it is well-known from the Lie theory of differential equations that unrestricted transformations will lead to an infinite number of generators which cannot be determined systematically.

The two problems which were chosen for this demonstration are well-known for their properties and their usefulness in illustrating theory in mechanics. The oscillator is the paradigm of linear systems and the Kepler problem the simplest of the important non-linear systems. In using these well-worked problems we have, even still, been able to make a point which, when made, is obvious, but has not, to our knowledge, been made. The classical invariants which are used to 
determine the equations of the orbits, energy, angular momentum and JauchHill-Fradkin tensor/Runge-Lenz vector, may be found, in the Lie approach, from the generator of time translations. Since, in Hamiltonian mechanics, the Hamiltonian is the generator of time translations for time-independent quantities, any such quantity which has zero Poisson bracket with the Hamiltonian is an invariant under time translation. That the same invariant arises from more than one generator is not exceptional as, in the case of the free particle in one-dimension, eight generators give rise to only three linearly independent invariants when the Lie method is used.

The Lie method yields more than Noether's theorem, under the same type of permitted transformations, for two reasons. The first is simply the possibility of obtaining more generators. The second is that, for a given generator, the equations of motion enter into the determination of possible invariants. We saw, especially in dealing with the $\partial / \partial t$ generator, that this permits manipulations which are not possible with Noether's theorem since, for the latter, the relationship between generator and invariant is predetermined.

In examining a particular problem with a view to establishing its orbit characteristics, we are not interested in every possible invariant, but only those which are of use in describing the orbit. Of the many invariants associated with the harmonic oscillator only the energy, angular momentum and Jauch-HillFradkin tensor are used for this purpose. For the Kepler problem it happens that all the invariants found are of this type and this may well be a feature of non-linear systems. It has been noted elsewhere that linear systems are special [9]. The Lie method does involve some further manipulation which could, in some problems, depend upon the ingenuity of the investigator to determine the invariants. The beauty of Noether's theorem is that the theory does not require further ingenuity once the generators are known. The failure of Noether's theorem is that it does not produce all the known orbit-determining invariants. If Noether's theorem could be suitably generalized so that all of these invariants are available, its utility would be greatly enhanced. For reasons already mentioned, velocity-dependent transformations are not suitable. The question is this, is there some other way to generalize the theorem to produce the desired results?

\section{Appendix}

In the Introduction, the use of Noether's theorem with point transformations was criticized on the gounds that it did not give rise to all of the known first integrals. In particular, for the problems under consideration here, Noether's theorem does not yield the Jauch-Hill-Fradkin tensor for the oscillator on the 
Runge-Lenz vector for the Kepler problem. Those first integrals may be obtained if velocity-dependent transformations are admitted, the latter being given as an example by Levy-Le Blond [4] and also in the text by Saletan and Cromer [8]. However, the reader will recall that the introduction of velocity-dependent transformations was also criticized in the Introduction as it gave rise to an infinite number of generators of symmetry transformations for which there is no systematic method of determination. The apparent contradiction in the last two sentences is readily resolved. In the paper and text cited, the authors do not derive their generators, but simple quote them and demonstrate that the Runge-Lenz vector follows. The concern of the present paper is the determination of generators. The problems of finding velocity-dependent transformations are demonstrated explicitly below.

Suppose that the Action Integral

$$
J=\int_{t_{0}}^{t_{1}}\{\mathbf{p} \cdot \dot{\mathbf{q}}-H(\mathbf{q}, \mathbf{p}, t)\} d t
$$

is invariant under the infinitesimal transformation generated by

$$
Y(\mathbf{q}, \mathbf{p}, t)=\xi(\mathbf{q}, \mathbf{p}, t) \partial / \partial t+\boldsymbol{\eta}(\mathbf{q}, \mathbf{p}, t) \cdot \partial / \partial \mathbf{q}+\zeta(\mathbf{q}, \mathbf{p}, t) \cdot \partial / \partial \mathbf{p} .
$$

Then, from Noether's theorem, there exists a first integral

$$
I(\mathbf{q}, \mathbf{p}, t) \doteq \mathbf{p} \cdot \eta(\mathbf{q}, \mathbf{p}, t)-H(\mathbf{q}, \mathbf{p}, t) \xi(\mathbf{q}, \mathbf{p}, t)-f(\mathbf{q}, t),
$$

where $f(\mathbf{q}, t)$ is the gauge-variant contribution. Note that the exclusion of $\mathbf{p}$ from $f$ does not indicate any loss of generality. To determine the generator, (A3) is differentiated with respect to time and set equal to zero. This gives rise to the equation

$$
\begin{aligned}
\mathbf{p} \cdot\left(\frac{\partial \eta}{\partial t}+[\eta, H]_{P B}\right) & -\frac{\partial H}{\partial \boldsymbol{q}} \cdot \boldsymbol{\eta}-\frac{\partial H}{\partial t} \xi-H\left(\frac{\partial \xi}{\partial t}+[\xi, H]_{P B}\right) \\
& -\frac{\partial f}{\partial t}-[f, H]_{P B}=0 .
\end{aligned}
$$

In view of the fact that $\xi$ and $\eta$ have been assumed to contain p, (A4) cannot be separated by coefficients of powers of $p_{i}$ as before. This is the basis for the remark made above that the generators cannot be determined systematically when velocity (momentum)-transformations are admitted.

It is, of course, possible to postulate forms for $\xi(\mathbf{q}, \mathbf{p}, t)$ and $\eta(q, \mathbf{p}, t)$ as polynomials in $\mathbf{p}$ with undetermined coefficients, substitute these and determine the coefficients. However, this procedure provides no guarantee of completeness in the set of operators. In the case of the Kepler problem, for example, it may be verified by direct substitution in (A4) that an operator is the generator of a symmetry transformation, which is the case of the example given by Saletan and Cromer ([8], page 82). 
It will be recalled that the use of velocity dependent transformations was also criticized on the grounds that there exist in infinite number of them. This is a well-known consequence of Lie's theory of differential equations, but may be demonstrated in a very trivial fashion. Suppose that it has been shown that

$$
Y(\mathbf{q}, \mathbf{p}, t)=\xi(\mathbf{q}, \mathbf{p}, t) \frac{\partial}{\partial t}+\eta(\mathbf{q}, \mathbf{p}, t) \cdot \frac{\partial}{\partial \mathbf{q}}+\zeta(\mathbf{q}, \mathbf{p}, t) \cdot \frac{\partial}{\partial \mathbf{p}}
$$

is a generator of a symmetry transformation. Then, if $G(I)$ is an arbitrary function of invariants (denoted generically by $I$ ),

$$
\tilde{Y}(\mathbf{q}, \mathbf{p}, t)=G(I) Y(\mathbf{q}, \mathbf{p}, t)
$$

is also a generator. This follows from direct substitution in (A4). As an infinite number of such functions $G(I)$ exists, there is an infinite number of velocity-dependent generators. For, even if the original $Y$ were the generator of a point transformation, its derivatives $\tilde{Y}$ must be velocity-dependent since first integrals are functions of $\mathbf{p}$ as well as of $q$ (and $t$ ).

In this Appendix it has been shown that Noether's theorem does have disadvantages when velocity-dependent transformations are admitted. The basic cause is very practical. How are generators to be found if (A4) cannot be solved? This difficulty with Noether's theorem is compounded by the fact that, without velocity-dependent transformations, it provides an incomplete set. For those two reasons it is suggested that the method of the Lie theory of extended groups, based on point transformations, is more satisfactory.

\section{References}

[1] D. S. Djukic, "A contribution to the generalized Noether's theorem", Arch. Mech. Stos. 26 (1974), 243-249.

[2] P. G. L. Leach, "The complete symmetry group of a forced harmonic oscillator", J. Austral. Math. Soc. B 22 (1980), 12-21.

[3] P. G. L. Leach, "The complete symmetry group of the one-dimensional time-dependent harmonic oscillator", J. Math. Phys. 21 (1980), 300-304.

[4] J. M. Levy-Leblond, "Conservation laws for gauge-invariant Lagrangians in classical mechanics", Amer. J. Phys. 39 (1971), 502-506.

[5] G. E. Prince and C. J. Eliezer, "Symmetries of the time-dependent $N$-dimensional oscillator", J. Phys. A 13 (1980), 815-823.

[6] G. E. Prince and C. J. Eliezer, "On the Lie symmetries of the classical Kepler problem", Research Report AM79:06 (Department of Applied Mathematics, La Trobe University).

[7] G. E. Prince and P. G. L. Leach, "The Lie theory of extended groups in Hamiltonian mechanics", Hadronic J. 3 (1980), 941-961.

[8] E. J. Saletan and A. H. Cromer, Theoretical mechanics (Wiley, New York, 1971), 82-83. 
[9] W. Sarlet and L. Y. Bahar, "A direct construction of first integrals for certain non-linear dynamical systems", reprint (Instituut voor Theoretische Mechanica, Rijksuniversiteit, Gent, B-9000 Gent, Belgium).

Department of Applied Mathematics

La Trobe University

Bundoora

Victoria 3083 